\title{
Stochastic Planning of Distribution Lines
}

\author{
A. Sauhats, L. Petrichenko, S. Beryozkina \\ Institute of Power Engineering \\ Riga Technical University (RTU) \\ Riga, Latvia \\ sauhatas@eef.rtu.lv, lubova.petricenko@rtu.lv, \\ svetlana.berjozkina@gmail.com
}

\author{
N. Jankovskis \\ Department of New Connection function \\ JSC "Latvenergo" \\ Riga, Latvia \\ nauris.jankovskis@latvenergo.lv
}

\begin{abstract}
The distribution line planning task has become more complex due to several impacting factors. This paper presents a stochastic approach for implementing an optimization procedure with a statistical representation of the electricity prices, ambient temperature and load currents in the distribution network. The validation of this stochastic approach is based on the comparison results of both examined approaches (stochastic and deterministic), which are applied for the computation of the total average annual costs. Attention has been focused on reducing total average annual costs by optimizing the conductor profile of a power line and the transformer capacity. Thus, the Net Present Value of the total average annual costs can be evaluated during the planning period. The problem is formulated as a minimization of the Net Present Value of the total average annual costs of an examined distribution line project. The tool is implemented in MATLAB software by using the Monte Carlo method. The proposed stochastic approach has been applied in the solution of an example of a distribution line project, which is presented.
\end{abstract}

Index Terms--Costs, power distribution line, optimization, planning

\section{ABBREVIATION}

$\begin{array}{ll}\text { DL } & \text { Distribution line } \\ \text { DLP } & \text { Distribution line planning } \\ \text { DA } & \text { Deterministic approach } \\ \text { MC } & \text { Monte Carlo } \\ \text { NPV } & \text { Net Present Value } \\ \text { PL } & \text { Power line } \\ \text { SA } & \text { Stochastic approach } \\ \text { TS } & \text { Transformer substation } \\ \text { TAAC } & \text { Total average annual costs }\end{array}$

\section{INTRODUCTION}

During the recent years, power industries have been facing significant changes, which have been caused by deregulation and liberalization of the energy markets, an increase in the installed capacity of renewable energy sources, the growing cost of energy, etc. These factors may result in a necessity to build new distribution lines (DLs) or restore the existing ones and to look for more efficient distribution line planning (DLP) methods and techniques [1-3]. Thus, the important subtask of economic DLP optimization can be stated as minimization of the total average annual costs (TAAC) (which include construction and operation costs) or maximization of the future expected profits. This target can be achieved by considering the capital investments, power losses, operation and maintenance costs, voltage losses, etc. taking into account the random nature of energy price, load power, and climate conditions.

In a deregulated electricity market, the dynamic and stochastic nature of planning and design environments must be taken into account, causing a more complex formulation of the task due to the large number of state variables (energy price, load power, climate conditions) and decision variables - DL parameters (conductor type and cross-section, tower type and coordinates, line accessories, etc.), the transformer capacity as well as the random and uncertain character of some variables (energy price, load power, meteorological conditions). The numerous line design standards, limitations and requirements must also be taken into account.

Influenced by stochastic processes and uncertain factors, the annual costs of DLs should be considered as a random variable. The optimization problem can be stated as a task of minimizing the Net Present Value (NPV) [4] of the average costs during a planning period. If needed, the risks can also additionally be assessed [5].

Before choosing exact line parameters, the following important tasks need to be solved:

1. A decision has to be made regarding the construction of the line, its route, voltage level, and power. In the general case, a new line influences the operation of the whole network and the connected generators and the above-mentioned tasks are solved by using wide area network models [6-7].

2 . The parameters (alternatives) of the line design have to be chosen, for example, conductor type and cross-section, tower type and coordinates, line accessories, etc.

The present study is only limited to the second task.

When comparing the line design alternatives, it is necessary to choose the criterion for the evaluation of their efficiency. Considering that all the compared alternatives are capable of meeting the energy demand of the consumers, it can be assumed that the revenues are equal in all the considered cases. As a result, the alternative that ensures the lowest project TAAC is to be considered the most favorable 
one during the particular evaluation period. Thus, the optimization task in this study is formulated as minimization of the NPV [8] of the TAAC. As a result, the best DL design solution with the minimum NPV of the TAAC can be found.

Several methodologies have been proposed to solve the DLP task. In general, there are two basic approaches: the deterministic method and the stochastic one. Most of the existing known deterministic approaches (DAs) are out-ofdate and/or need modifications and improvements due to not taking into account the variations of energy price, ambient temperature, and load current that consequently lead to higher values of DL costs. The most promising directions of current research into efficient planning of future grids are focused on the implementation of stochastic approaches (SAs).

In this way, the greatest difficulties are related to the need for a large amount of input information. For collecting the required data, the smart grid technologies (for example, a geographic information system (GIS) and smart metering systems) can be implemented since it is possible to perform multidimensional registration of impacting parameters: load power, ambient temperature, electricity prices, etc. Registration records can be considered as realizations of random processes. The results of load power records can be collected in an electronic library, which contains processes for different consumer types. There is an opportunity of predicting power demand in terms of a certain set of consumers. The information on the planned consumer connections can be obtained from this library taking into account development plans of the cities and regions that are under study.

The most important contribution of this paper is the proposed SA for DLP optimization applying historical records of electrical loads, ambient temperature and electricity prices (see Section V). Based on the essence of the proposed SA, computation software is developed. The proposed optimization model minimizes the TAAC. The tool is implemented in MATLAB software by using the Monte Carlo (MC) method. The second contribution is a comparison of the estimation results of the NPV of the TAAC of DL projects obtained by applying both deterministic and stochastic approaches during the evaluation planning period. A validation of the considered approaches is implemented based on the data of a real-life case study.

The paper is organized as follows: Section I explains the abbreviations that have been used in the paper, Section II is devoted to the topicality of the problem. Section III describes the mathematical formulation of the optimization problem as well as represents the key issues of the deterministic approach and the stochastic approach. Section IV reviews the load study. Section V introduces a case study with the obtained results. Finally, the conclusions will be outlined in Section VI.

\section{THE THEORETICAL BACKGROUND}

Let us assume that the initial construction costs of every alternative $j$ are equal to $C_{o j}$ whereas over year $t$ the costs are equal to $C_{t j}$. By using the defined quantities, the NPV of the TAAC of the alternatives for the end of the planning period can be calculated as follows:

$$
N P V_{j}=C_{o j}+\sum_{t=1}^{N} \frac{C_{t j}}{(1+i)^{t}},
$$

where: $i$ - rate of return; $N$ is the evaluation planning period (EP), years; and the alternative with the lowest planning period TAAC can be selected.

\section{A. The Deterministic Aproach (DA)}

The DA neglects the random and uncertain character of variables. The TAAC of the single line in year $t$ can be defined as follows [9]:

$$
\begin{aligned}
& C_{s}=\left(i+p_{\Sigma L}\right) \cdot K_{L} \cdot l+\frac{P^{2}}{U^{2} \cdot \cos \phi^{2}} \cdot R_{o} \cdot l \cdot \beta \cdot 10^{-3}+ \\
& +\left(i+p_{\Sigma T}\right) \cdot K_{T}+\Delta A_{S} \cdot \beta, \\
& \Delta A_{S}=\Delta P_{t g, t} \cdot T+\Delta P_{v} \cdot\left(\frac{S_{\max }}{S_{n T}}\right)^{2} \cdot \tau,
\end{aligned}
$$

where $i$ is the market interest rate (interest on loan), r.u.; $p_{\Sigma L}$ and $p_{\Sigma T}$ are the total deductions on depreciation, routine repair and maintenance from the capital investments in the DL and transformer substation (TS), respectively, r.u.; $K_{L}$ and $K_{T}$ stand for the DL and TS capital investments, $€ ; l$ is the length of $\mathrm{DL}, \mathrm{km} ; R_{0}$ is the resistance of the conductor of the line, $\Omega / \mathrm{km} ; \beta$ is the specific price of the electric energy losses, $€ / \mathrm{kWh} ; \Delta A_{S}$ stands for the active energy losses, $\mathrm{kWh} ; \tau$ is the utilization time of maximum losses per year, $\mathrm{h} ; T$ - hours per year, $\mathrm{h} ; \Delta P_{\mathrm{tg}, t}$ - the no-load losses in the transformer, $\mathrm{kW} ; \Delta P_{v}$ - the short circuit losses in the transformer, $\mathrm{kW}$; $S_{\text {max }}$ - the actual maximum load of the TS, kVA; $S_{n T}$ - the nominal power of the transformer, kVA [9].

The relationship between the costs and the line power is a nonlinear function, as we can see. If the parameters (electricity prices, ambient temperature and load currents (power)) are assumed to not be random, then the DA can be used. However, it can be stated that the dispersions of the load current, the electricity prices, and the line resistances (considering the temperature dependence) are considerable and influence the final calculation results. The variability of the line power in (3) is taken into account by choosing the annual utilization period $\tau$ (the accuracy of this value is questionable under market conditions); the price variation is not taken into account at all. Therefore, it becomes necessary to investigate the suitability of the DA or the need to replace it with the more accurate stochastic approach.

\section{B. The Stochastic Approach (SA)}

Analyzing (2) and (3), one can claim that the line load, the costs of the power losses and the temperature are random time-dependent parameters, which are correlated to one another. Correspondingly, also $C t$ is a random time function.

The multidimensional random function $C t$ can be simplified by discretization to a number of time periods $\left(t_{1}\right.$, $\left.t_{2} \ldots t_{n}\right)$. A probability distribution function $\varphi$ can be assigned to each time period [10]. Complex and extensive calculations are 
needed. The estimation of the average costs of the multidimensional random function $C t$ can be simplified and expressed as follows:

$$
\begin{aligned}
& C_{t j}=\sum_{T i}^{T i+8760}\left(\left(i+p_{\Sigma L}\right) \cdot K_{L} \cdot l+\frac{\mathbf{P}_{\text {stoch j }}{ }^{2}}{U^{2} \cdot \cos \phi^{2}} \cdot R_{\text {rat }} \cdot l \cdot \boldsymbol{\beta}_{\text {stoch } j} \cdot 10^{-3}+\right. \\
& \left.+\left(i+p_{\Sigma T}\right) \cdot K_{T}+\Delta A_{S} \cdot \boldsymbol{\beta}_{\text {stoch } j}\right) \\
& \Delta A_{S}=\Delta P_{t g, t}+\Delta P_{v}\left(\frac{\mathbf{P}_{\text {stoch } j}}{S_{n T} \cdot \cos \phi}\right)^{2}, \\
& R_{\text {rat }}=R_{0} \cdot\left(1+a_{\text {tem }} \cdot\left(\mathrm{T}_{\text {amb.stoch } j}-20\right)\right)
\end{aligned}
$$

where $T i-T i+8760$ is the duration of the period, which is equal to one year; $R_{\text {rat }}$ is the rated resistance of the conductor of the line, $\Omega / \mathrm{km} ; P_{\text {stoch }}$ is a random load line's value, $\mathrm{kW}$; $\beta_{\text {stoch }}$ is a random specific price of the electric energy losses value, $€ / \mathrm{kWh} ; a_{\text {tem }}$ is the temperature coefficient, $1 / \mathrm{K}$; $T_{a m b . s t o c h}$ is a random value of ambient temperature, $\mathrm{C}^{\circ}$.

Using (4) and MC method allows us to compute the average value of annual costs based on a multidimensional random process with $P_{\text {load }}, \beta, T_{a m b}$. Thus, the TAAC are calculated by using extrapolated and modified data. Due to the implementation of new smart grid technologies, realization of (4) becomes simple and makes it possible to perform multidimensional registration of parameters (line load, ambient temperatures, prices, etc.) and to create an electronic database of required statistical data (an electronic library). Extensive calculations can be simplified by using the MC method [11], which is applicable for calculations of the average costs (4) in the particular optimization task. To implement the MC method, modeling of random processes has to be performed; in this way, a library of random processes has been created and used, from which the values of the above-mentioned random time-dependent parameters can be chosen. The presented SA is implemented in software and enables quick and easy solution of the set optimization task. The solution is realized in MATLAB.

A detailed description of the SA is given in [12].

\section{THE IMPLEMENTATION OF STATISTICAL DATABASES}

Electric power networks are characterized by a multifaceted composition of consumers: dwelling houses, industrial, public utility and services enterprises, electrified city and intercity transportation and others. Various groups of consumers significantly differ in terms of consumer capacity, operating modes, requirements regarding the quality of electric power and uninterrupted operation of power supply $[13,14]$. Precise definition of the total prospective electrical load of the region is an important issue, which must be taken into account during the DLP.

A DA to the determination of the total electrical load is widely used in the practice of electrical design companies. Such an approach is based on the estimated specific load for each consumer, taking into account the simultaneity coefficient. The above-mentioned parameters are reference data [15].
The application of the proposed SA becomes simple due to implementation of smart grid technologies, which allow performing multidimensional registration of such parameters as power, ambient temperature, electricity prices, etc. As a result, it becomes possible to create an electronic library of these statistical data. For predicting the ambient temperature and electricity price, the statistical database of the market operator (here — NordPool [16] and the meteorological service (for Latvia - [17]) can be used accordingly.

The library creation process takes into account a number of factors that significantly affect the overall scene of electricity consumption and its prices. Workloads of different consumer groups, for example, multi-family residential buildings, stores, hospitals, public buildings, plants and factories, office buildings, private, generation substations, are taken as the basis of the library establishment process. The predicted load of PL here is presented as random process realizations, which are determined by summarizing every hour the workloads of different consumer groups. The existence of such an electronic library of electricity consumption processes depending on different consumer groups makes it possible to predict the total DL loading based on the region development plans by simply adding the workload of such consumers to the existing plan of the region or city. Prediction can be performed using an artificial neural network [18]. The results based on a specific case study are presented in Section V.

\section{The OBTAINED RESUltS AND ANALYSIS}

A simple case study is considered, when there is a $20 \mathrm{kV}$ PL and a $110 / 20 \mathrm{kV}$ transformer. The DL alternative design conditions are assumed based on the existing data of a line and a substation of the Latvian distribution network.

The estimation algorithm that has been used consists of three main stages that are described below:

1) Prediction of the total electrical load of the prospective region, taking into account the scenarios for load growth as well as its planning and development aspects;

2) Selection of the optimal variant of the conductor crosssection of a DL with minimum TAAC including computation of capital costs as well as estimation of power losses and checking of the selected conductor regarding the voltage losses;

3) Computation and analysis of the NPV of the TAAC for both examined methods (SA and DA) during the evalution planning period. Selection of the best project variant by selecting the optimal DL parameters, namely, conductor profile as well as transformer capacity.

\section{A. The First Stage}

Forecasting a future DL power demand implies prediction of the load increase taking into account its variable nature. Load changes cause power losses in a DL. The cost of these losses greatly depends on the electricity price, which varies constantly, as well as the ambient temperature. Since the power records of the existing substation are available (a statistical database is used), its real-time consumption as well as the users' load data can be found. The examined substation 
service area includes a total number of objects that exceeds 10000 consumers. The statistical data was defined from the Automatic Energy Accounting System (AEMS), the power consumed during the year - in terms of hours, $\mathrm{kWh}$. In the presented case study it is assumed that load power grows by $2 \%$ each year (the planning period equals 30 years).

\section{B. The Second Stage}

In the first part of the presented program, a detailed stochastic calculation and an assessment of load power, electricity price and ambient temperature of current year is made (the $1^{\text {st }}$ year of project implementation), using the $\mathrm{MC}$ method. This part includes the calculation of the TAAC (for one year) for all the project variants by the exhaustive search method. In general, the capital costs can be found in two ways: firstly, the accumulated experience can be used in PL design and construction - the average cost of line per $1 \mathrm{~km}$ is defined; secondly, powerful software, for example, PLSCADD, can be used. The tool contains the so-called "Project Estimator" module, which provides accurate calculation of the capital investments of the examined PL design alternative [19]. As a result, the optimal conductor cross-section of the DL is selected and checked regarding voltage losses.

Let us describe in detail the choice of optimal conductor cross-section for each transformer variant. It consists of two cycles: the internal cycle and the external cycle. The essence of the internal cycle is the following: the TAAC are calculated for each cross-section, using the record databases, which are inserted into the program automatically, and the number of trials (in our case the number of trials equals 1000). Within the external cycle, the minimum costs from all the TAAC are selected, and hence, corresponding to the minimum costs, the optimum cross-section is determined.

Fig. 1 and Fig. 2 show the results of each case according to the described SA.

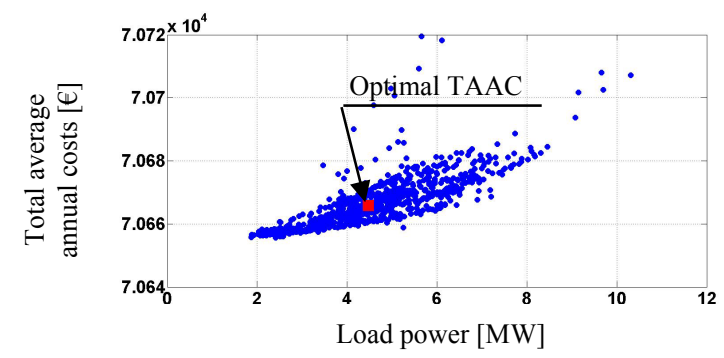

Figure 1. The calculated TAAC of line conductor AS-50 depending on load power (for the $1^{\text {st }}$ transformer).

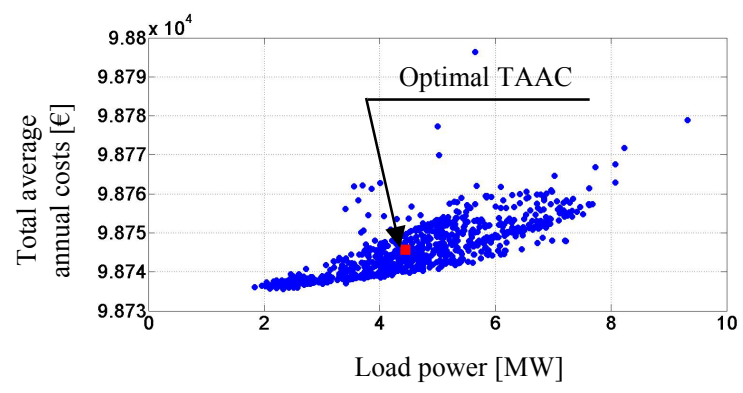

Figure 2. The calculated TAAC of line conductor AS-50 depending on load power (for the $2^{\text {nd }}$ transformer).
In our case study, the mentioned program calculated the TAAC of the $1^{\text {st }}$ year of project implementation. From six alternative variants of line cross-section, the first one (AS-50) has the lowest TAAC. Consequently, the graphs presented above show all the possible TAAC values of the selected line cross-section. As a result, the optimal (mean) TAAC value of AS-50 is determined: in the first case (for the $1^{\text {st }}$ transformer) the rated TAAC value is $70670 €$, in the second case (for the $2^{\text {nd }}$ transformer) $-98750 €$.

\section{The Third Stage}

In the second part of the above-described program, the NPV of TAAC is calculated for each year of the planning period. The estimations are based on the obtained results of the first part program's parameters ( $P_{\text {aver.stoch }}, \beta_{\text {aver.stoch }}$, $T_{\text {amb.aver.stoch, }} C_{\text {stoch }}$.

The obtained results and analysis for both approaches is presented in Fig. 3 - Fig. 6 and in Table I.

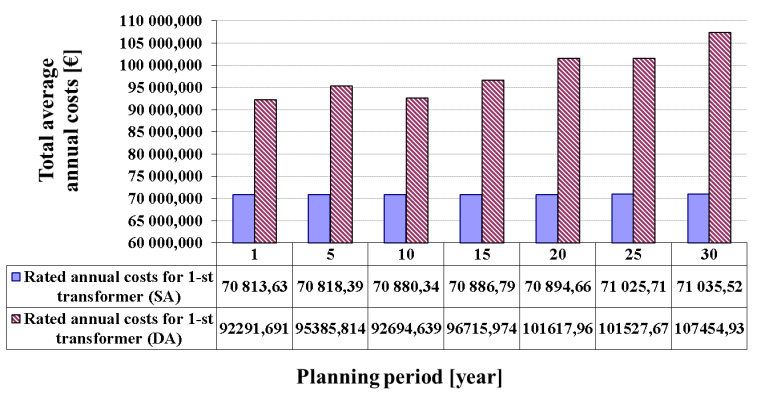

Figure 3. The calculated TAAC by DA and SA for the planning period (for the $1^{\text {st }}$ transformer)

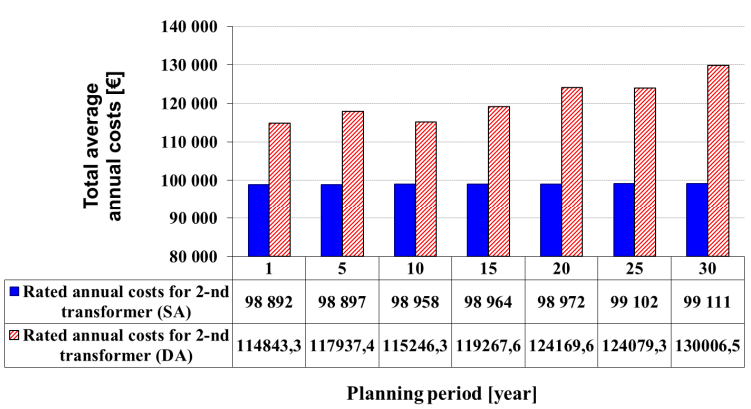

Figure 4. The calculated TAAC by DA and SA for the planning period (for the $2^{\text {nd }}$ transformer)

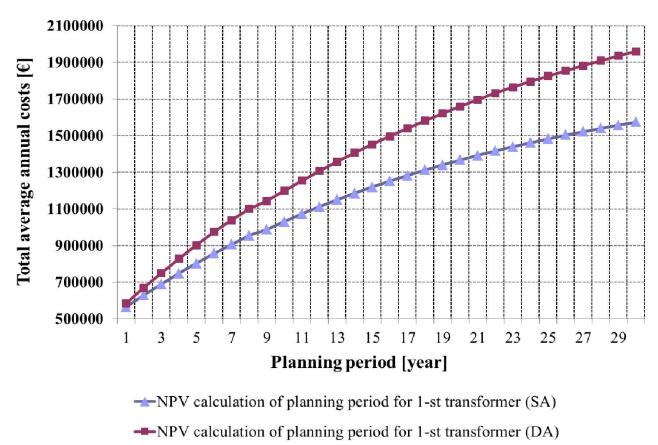

Figure 5. The calculated NPV of the TAAC by DA and SA for the planning period (for the $1^{\text {st }}$ transformer) 


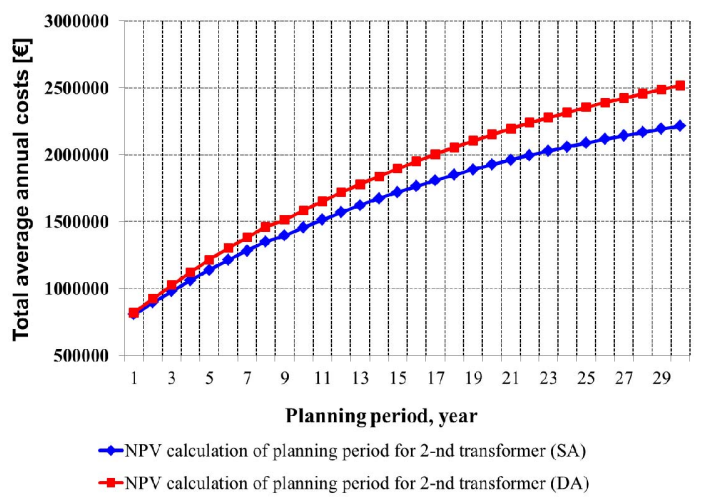

Figure 6. The calculated NPV of the TAAC by DA and SA for the planning period (for the $2^{\text {nd }}$ transformer)

TABLE I. COMPARISON RESULTS OF TwO APPLIED APPROACHES

\begin{tabular}{|c|c|c|c|}
\hline & SA & DA & $\begin{array}{c}\text { Difference, } \\
\mathbf{\%}\end{array}$ \\
\hline $\begin{array}{c}\text { Sum of rated } \\
\text { TAAC, } € \\
\left(1^{\text {st }} \text { transformer }\right)\end{array}$ & 2127316 & 2938016 & $\mathbf{3 8 , 1 0}$ \\
\hline $\begin{array}{c}\text { Sum of rated } \\
\text { TAAC, } € \\
\left(2^{\text {nd }} \text { transformer }\right)\end{array}$ & 2969634 & 3614564 & $\mathbf{2 1 , 7 1}$ \\
\hline $\begin{array}{c}\text { NPV of TAAC, } € \\
\left(1^{\text {st }} \text { transformer }\right)\end{array}$ & 1573659,14 & 1960109,09 & $\mathbf{2 4 , 5 5}$ \\
\hline $\begin{array}{c}\text { NPV of TAAC, } € \\
\left(2^{\text {nd }} \text { transformer }\right)\end{array}$ & 2216492,09 & 2518936,5 & $\mathbf{1 3 , 6 4}$ \\
\hline
\end{tabular}

As a result, there is a significant difference of TAAC values in the two approaches $(21 \%-39 \%)$. This fact can be justified mainly by the use of the parameters of annual utilization period and utilization time of maximum load in the DA method. Accordingly, differences in the NPV of the TAAC are also high: between $13 \%-25 \%$ (depending on selected transformer type). As a result, the implementation of SA provides a great opportunity to save thousands of hundreds of euros, which is an important amount for power engineering and the economy as a whole. Finally, it can be concluded that for all variants of the DL project, the presented SA demonstrates the validity and need of being used. Thus, the fluctuations of energy price as well as load (the capability of meeting the energy demand of the consumers) must be taken into account. These parameters play an important role and have a great impact on DLP, especially at market conditions.

\section{CONCLUSIONS}

1. The restructuring of the power systems and the use of market mechanisms essentially change the formulation of the task of substantiation and optimization of energy facility projects, since decisions are to be made at conditions of wide fluctuations of prices.

2. The traditional methods of solving the development optimization task that have been developed earlier have become outdated. Changes and verifications regarding the suitability of the traditional and existing approaches as well as searches for new appropriate methods are needed.
3. The SA is proposed and an algorithm has to be developed, and after the creation of appropriate software suitable for designers, it has to be put into practice.

4. A significant costs difference in absolute numbers equals thousands of hundreds of euros, which is certainly an important amount for a power system and the economy as a whole.

\section{ACKNOWLEDGMENT}

The work presented in this paper has been LATENERGI co-financed by the National Research Program LATENERGI (2014-2017).

\section{REFERENCES}

[1] V. Neimane, "On development planning of electricity distribution networks," Ph.D. dissertation, Dept. of Elec. Eng., Royal Institute of Technology, Stockholm, 2001.

[2] F. Kiessling, P. Nefzger, J.F. Nolasco, U. Kaintzyk, Overhead Power Lines Planning, Design, Construction, Springer-Verlag Berlin Heidelberg New York: 2003, p. 759.

[3] Zemīte, L., Gerhards, J., Gorobecs, M., L,evčenkovs, A. Optimization of Distribution Systems Reliability with the Stochastic Behavior. In: 2015 56th International Scientific Conference on Power and Electrical Engineering of Riga Technical University (RTUCON): Proceedings, Latvia, Rīga, 14-16 October, 2015.

[4] A. Al-Hinai, A. Al-Badi, E. A. Feilat, M. Albadi. "Efficiency Enhancements of Electric Power System and Economic AnalysisPractical Case Study", Int. J. of Thermal \& Environmental Engineering Volume 5, No. 2 (2013) pp. 183-190.

[5] M. Shahidehpour, H. Yamin, Z. Li, Market Operations in Electric Power Systems: Forecasting, Scheduling and Risk Management. New York: Wiley-Interscience, 2002, p. 531.

[6] C. Langhorne, C. Carlson and S. Chowdhury, "Wide area network performance modeling of distributed energy management system," IEEE Transactions on Power Systems, vol. 9, 1994, pp. 730-735.

[7] Yi Deng, Hua Lin, S. Shukla, J. Thorp, L. Mili, "Co-Simulating Power Systems and Communication Network for Accurate Modeling and Simulation of PMU based Wide Area Measurement Systems using a Global Event Scheduling Technique", in Proc. 2013 Modeling and Simulation of Cyber-Physical Energy Systems (MSCPES), pp. 1-6.

[8] P. L. Anderson, "The economics of business valuation. Towards a value functional approach", Stanford, California: Stanford Economics and Finance, an imprint of Stanford University Press: 2012, p. 424.

[9] A. Vanags, Z. Krishans. Electrical network and systems, II part, 2005. g., RTU publisher. (in Latvian)

[10] G. Korn, T. Korn, Mathematical handbook for scientists and engineers, 4-th enlarged and revised ed., Moscow: 1978, p. 832 (in Russian).

[11] G. S. Fishman, Monte Carlo: Concepts, Algorithms, and Applications, Springer-Verlag New York: 1995, p. 682.

[12] A. Sauhats, S. Beryozkina, L. Petrichenko, V. Neimane, "Stochastic optimization of power line design," in Proc. IEEE PES Eindhoven PowerTech2015, pp. 892-897.

[13] J. Dickert, P. Schegner, "Residential load models for network planning purposes", in Proc. 2010 Modern Eletric power Systems (MEPS), p. 6.

[14] S. Heunis and R. Herman, "A probabilistic model for residential consumer loads," IEEE Transactions on Power Systems, vol. 17, 2002, pp. 621-625.

[15] Edited by D. Faybisovich, Electric power networks designing manual, 2nd edition, Moscow, NC ENAS Publishing House: 2006, p. 102.

[16] Nord Pool Spot database. [Online]. Available: http://www.nordpoolspot.com/

[17] Meteorology data in Latvia. [Online]. Available: http://www.meteoprog.lv

[18] B. Yegnanarayana, Artificial Neural Networks, Prentice-Hall, New Delhi, India: 2006, p. 461.

[19] PLS-CADD ${ }^{\text {TM }}$ (Power Line Systems-Computer Aided Design and Drafting) User's manual, 2011, p. 468.

This is a post-print of a paper published in Proceedings of the 2016 13th International Conference on the European Energy Market (EEM 2016) http://dx.doi.org/10.1109/EEM.2016.7521349 and is subject to IEEE copyright. 